\title{
Spectral Distortions of Metalloporphyrin Forms at Low Temperatures
}

A. S. Starukhin ${ }^{\mathrm{a}},{ }^{*}$, A. V. Gorski ${ }^{\mathrm{b}}$, and M. Z. Kijak ${ }^{\mathrm{b}}$

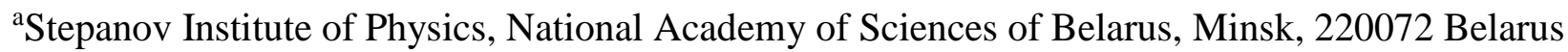

${ }^{\mathrm{b}}$ Institute of Physical Chemistry, Polish Academy of Sciences, Warsaw, 01-224 Poland

Bulletin of the Russian Academy of Sciences: Physics, 2018, Vol. 82, No. 12, pp. 1560-1564.

DOI: $10.3103 / \mathrm{S} 1062873818120225$

This project has received funding from the European Union's Horizon 2020 research and innovation programme under the Marie Skłodowska-Curie grant agreement No. 645628

Project Number: 645628

Project Acronym: METCOPH

Project title: Metallocomplexes of macrocyclic compounds

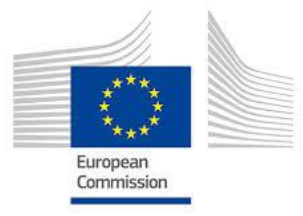




\title{
Spectral Distortions of Metalloporphyrin Forms at Low Temperatures
}

\author{
A. S. Starukhin ${ }^{\mathrm{a}}$ *, A. V. Gorski ${ }^{\mathrm{b}}$, and M. Z. Kijak ${ }^{\mathrm{b}}$

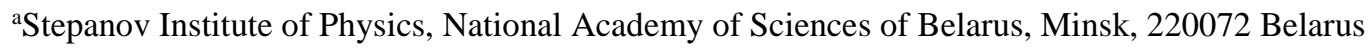 \\ ${ }^{\mathrm{b} I n s t i t u t e}$ of Physical Chemistry, Polish Academy of Sciences, Warsaw, 01-224 Poland \\ *e-mail: a.starukhin@ifanbel.bas-net.by
}

\section{ABSTRACT}

The planar form and two types of distorted molecular conformations in the ground electronic state for a number of metalloporphyrins in solid matrices exist simultaneously. A comparison of experimental data and results from quantum chemical calculations is discussed in order to analyze the structure of distorted porphyrin macrocycles at cryogenic temperatures.

\section{INTRODUCTION}

Molecules of porphyrins and metalloporphyrins are, along with their analogs, chromophores in such biologically important compounds as green plant chlorophyll and blood heme (see [1] and references therein). These compounds have high degrees of symmetry and a variety of spectral and photophysical characteristics, and are also good probes for studying many processes of intramolecular and intermolecular interactions. It is known that porphyrins and their metal complexes have planar structure and high degrees of symmetry in neutral solvents at room temperature $[1,2]$. However, distortions of the planar molecular structure of the porphyrin macrocycle are possible under certain conditions. This leads to spectral manifestations of the effects of nonplanarity for these compounds. Introducing various groups into different positions of the macrocycle (e.g., forming the twice protonated form of porphyrin [3, 4] and introducing alkyl groups in various combinations and into different positions of the macrocycle [5-7]) can distort the planar structure of the porphyrin macrocycle. The structure of metalloporphyrin is also distorted upon the extraliganding of the central metal atom $[8,9]$ and the disturbing effect of the crystal field for different types of crystal structure. 
The spectral, energetic, physical, and chemical properties of these compounds vary considerably, compared to planar tetrapyrrole macrocycles with the nonplanarity of the porphyrin macrocycle in the ground and excited states $[10,11]$. Distorted structures of metalloporphyrins are most often recorded and analyzed. Analysis of resonant Raman scattering (RRS) spectra of the metal complexes of porphyrins (with Ni, Pd, Fe, Zn, and other atoms) shows that lines in the $1500-1650 \mathrm{~cm}^{-1}$ region are the ones most shifted [12]. A comparison of the RRS spectra of metalloporphyrins with various substituents (e.g., alkyl and phenyl groups) and metal complexes of porphyrins with various metals makes it difficult to interpret experimental data.

One of the greatest causes of nonplanar distortions for metalloporphyrins [8,9] is the interaction between the metal complexes of porphyrins and extraligands. Raman spectroscopy is not selective enough to allow us to obtain information on the frequencies of normal vibrations of different spectral forms. In addition, complexes with ligands are normally less than stable at room temperature, and adding other organic solvents (e.g., ligands) to a solution makes the recorded spectra much more complicated. It is therefore harder to analyze experimental results.

The aim of this work was to study via low-temperature selective spectroscopy different spectral forms of the simplest metal complexes of porphin under conditions of their stabilization at helium temperatures.

\section{EXPERIMENTAL}

Spectral characteristics of metal complexes of porphin with $\mathrm{Mg}(\mathrm{II}), \mathrm{Zn}(\mathrm{II}), \mathrm{Pd}(\mathrm{II})$, and $\mathrm{Pt}(\mathrm{II})$ ions, and of analogous compounds with alkyl substituents on pyrrole rings and the corresponding ions, were recorded when these compounds were placed in a Shpol'skii matrix, a tetrahydrofuran matrix, and hard matrices of inert gases according to the procedure in [13].

Let us consider the effects of the nonplanarity of porphin metal complexes using mainly the example of the zinc complex of porphin $(\mathrm{ZnP})$, due the limited number of works. The fluorescence spectra for this compound in an $n$-octane matrix (Fig. 1a) and an $n$-octane matrix with the addition of around $1 \%$ pyridine (Fig. 1b) are shown in Fig. 1. 

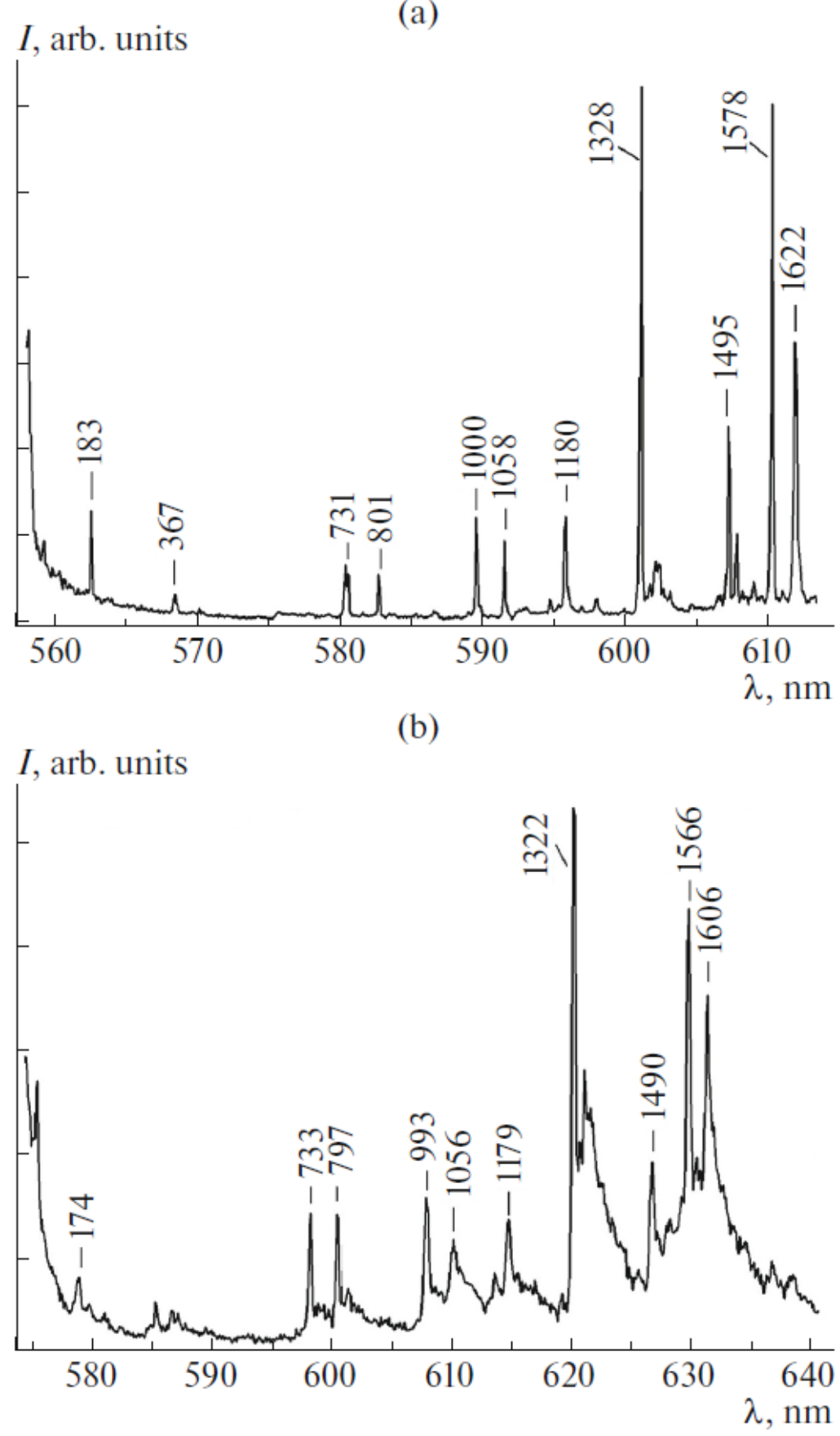

Fig. 1. Fluorescence spectra of (a) the $\mathrm{ZnP}$ F0 form in $n$-octane upon selective laser excitation $\left(\lambda_{\text {excit }}=\right.$ $556.8 \mathrm{~nm})$ and (b) the FII form in $n$-octane upon selective laser excitation $\left(\lambda_{\text {excit }}=573.1 \mathrm{~nm}\right)$ at $4.2 \mathrm{~K}$.

The spectra were recorded via selective laser excitation in the region of the $0-0$ band of the absorption spectrum at $4.2 \mathrm{~K}$. The absorption spectrum shifted to $16 \mathrm{~nm}$ and the relative intensities of the bands in the absorption and fluorescence spectra changed upon adding the pyridine. Figure 1 shows the differences between the frequencies of normal vibrations for the same substance in the same solvent with 
a small addition of pyridine. The greatest differences between the frequencies are in the range of 1500 $1650 \mathrm{~cm}^{-1}$. These results can be interpreted as the registration of various spectral forms of $\mathrm{ZnP}$ in different solid solutions, in analogy with the results of [9].

Analogous effects of the formation of different forms when $\mathrm{ZnP}$ molecules are placed in a hard matrix of argon (Ar) at a temperature of $5 \mathrm{~K}$ and different wavelengths of excitation are shown in Fig. 2.

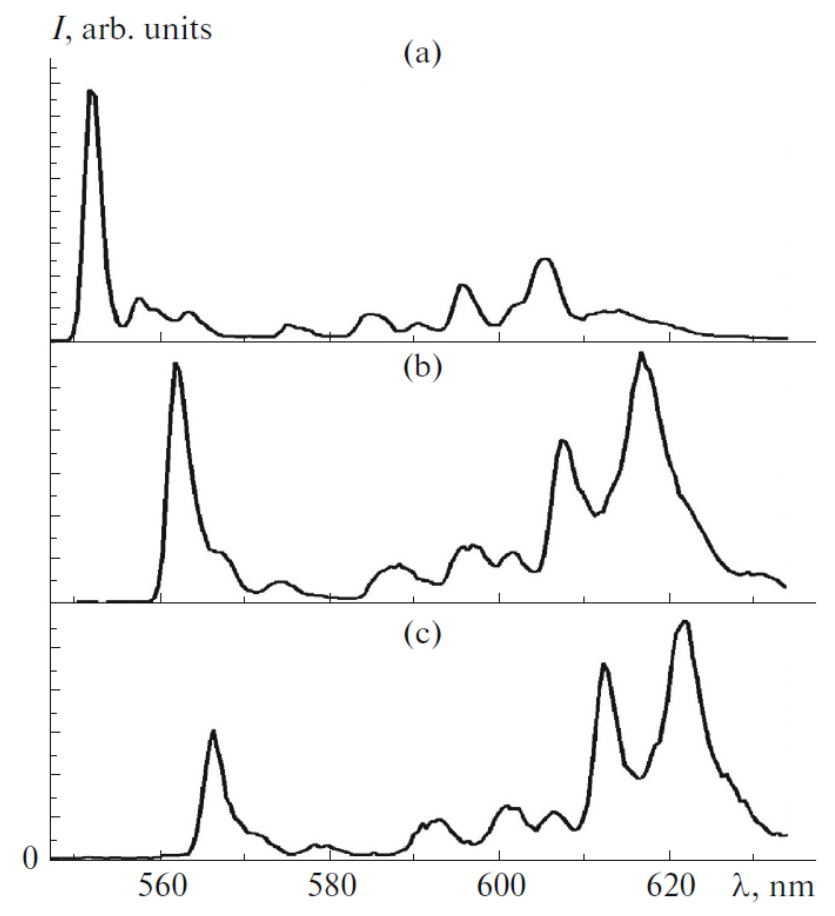

Fig. 2. Fluorescence spectra of $\mathrm{ZnP}$ condensed in a matrix of solid argon on a sapphire substrate upon nonselective excitation: (a) $\lambda_{\text {excit }}=385 \mathrm{~nm}$, (b) $\lambda_{\text {excit }}=388 \mathrm{~nm}$, and (c) $\lambda_{\text {excit }}=404 \mathrm{~nm}$ at $5 \mathrm{~K}$.

At least three different conformations of $\mathrm{ZnP}$ form in hard matrices. The set of experimental data (fluorescence spectra, fluorescence excitation spectra, behavior of bands in the region of the Soret band, and so on) confirms this conclusion. Data on the spectral characteristics of various spectral forms are given in Table 1. 
Table 1. Spectral characteristics of $\mathrm{ZnP}$ in hard argon matrices (Ar) and $\mathrm{THF}$ at helium temperatures

\begin{tabular}{l|l|l|l}
\hline \multirow{2}{*}{ Medium } & \multicolumn{3}{l}{ Positions of band maxima in absorption and fluorescence spectra } \\
\cline { 2 - 4 } & $\lambda_{\text {absorp }}\left(\mathrm{S}_{0} \rightarrow \mathrm{S}_{1}\right)$ & $\lambda_{\text {absorp }}($ Soret band $)$ & $\lambda_{\mathrm{fl}}$ \\
\hline Ar matrix & $552.3 \mathrm{~nm}$ & $384.7 \mathrm{~nm}$ & $552.3 \mathrm{~nm}$ \\
F0 form & $\left(18110 \mathrm{~cm}^{-1}\right)$ & $\left(25994 \mathrm{~cm}^{-1}\right)$ & $\left(18110 \mathrm{~cm}^{-1}\right)$ \\
\hline Ar matrix & $562.4 \mathrm{~nm}$ & $388.7 \mathrm{~nm}$ & $562.4 \mathrm{~nm}$ \\
FI form & $\left(17780 \mathrm{~cm}^{-1}\right)$ & $\left(25727 \mathrm{~cm}^{-1}\right)$ & $\left(17780 \mathrm{~cm}^{-1}\right)$ \\
\hline Ar matrix & $566.5 \mathrm{~nm}$ & $404.0 \mathrm{~nm}$ & $566.5 \mathrm{~nm}$ \\
FII form & $\left(17652 \mathrm{~cm}^{-1}\right)$ & $\left(24752 \mathrm{~cm}^{-1}\right)$ & $\left(17652 \mathrm{~cm}^{-1}\right)$ \\
\hline THF & $562.7 \mathrm{~nm}$ & $401 \mathrm{~nm}$ & $562.7 \mathrm{~nm}$ \\
FI form & $\left(17749 \mathrm{~cm}^{-1}\right)$ & $\left(24938 \mathrm{~cm}^{-1}\right)$ & $\left(17749 \mathrm{~cm}^{-1}\right)$ \\
\hline THF & $569.1 \mathrm{~nm}$ & $407 \mathrm{~nm}$ & $569.1 \mathrm{~nm}$ \\
FII form & $\left(17571 \mathrm{~cm}^{-1}\right)$ & $\left(24570 \mathrm{~cm}^{-1}\right)$ & $\left(17571 \mathrm{~cm}^{-1}\right)$ \\
\hline
\end{tabular}

Ar is argon; THF is tetrahydrofuran.

Conformation with the $0-0$ transition position at $552 \mathrm{~nm}$ corresponds to a planar molecular structure (F0 form) [9]. The spectral form with the $0-0$ transition position at around $562 \mathrm{~nm}$ corresponds to the distorted form of a saddle-like (saddle) structure (FI form), and the spectral form with a maximum at around $569 \mathrm{~nm}$ corresponds to a dome-like (dome) conformation (FII form). The fluorescence spectra of the FI form are shifted to the long-wavelength region by $300 \mathrm{~cm}^{-1}$, while the spectra of the FII form are shifted by $450 \mathrm{~cm}^{-1}$ with re-spect to the spectrum of the planar molecular structure (F0 form).

Since the luminescent forms are in the ground state (SO state), the existence of the three spectral forms is not associated with the splitting of long-wavelength electronic transitions due to a reduction in the symmetry of the metalloporphyrin in the matrix at low temperatures.

Quantum chemical calculations for these metalloporphyrins were performed using the density functional theory with the CAM-B3LYP functional and the basic set of def2SVP for optimization of the molecular structure. The structures of all three possible conformations for metalloporphyrins are shown in Fig. 3. 
(a)

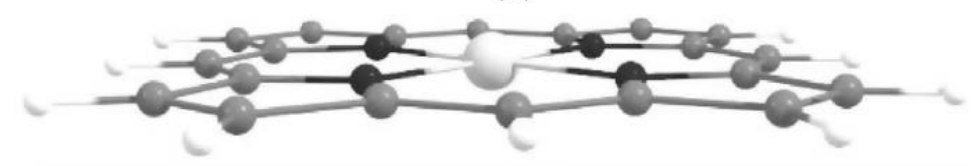

(b)

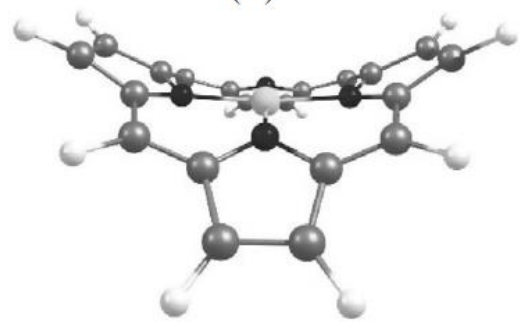

(c)

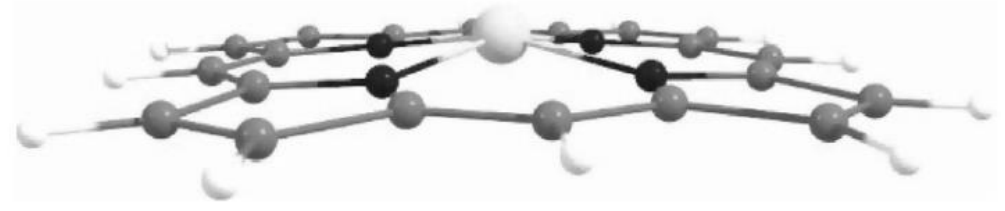

Fig. 3. Structures of molecular conformations of $\mathrm{ZnP}$, where (a) is a planar structure; (b) is a saddle structure, and (c) is a dome structure.

Structure 1 corresponds in particular to the planar conformation of $\mathrm{ZnP}$, while structure 3 corresponds to the dome conformation calculated for $\mathrm{ZnP}$ with an extraligand (the tetrahydrofuran molecule). Structure 2 was not calculated using the quantum chemical approach, but we assume a structure of this type is visible in the spectra as a saddle-conformation. The presence of such a structure was established using data on the frequencies of normal vibrations. Thin-structure spectra were recorded for all of these forms upon selective excitation into the $0-0$ transition band in the absorption spectrum. It was shown that the frequencies of the normal vibrations for the F0 and FI forms are virtually identical. A comparison of the frequencies of normal vibrations for the FI and FII forms showed that major differences are observed in the frequency range of $1400-1650 \mathrm{~cm}^{-1}$. As can be seen from the data in Fig. 1, lines with vibrations of about $1600 \mathrm{~cm}^{-1}$ are shifted the most. The lines corresponding to vibrations in the spectrum of the F0 form (Fig. 2) with frequencies of $1622 \mathrm{~cm}^{-1}$ are thus shifted by $16 \mathrm{~cm}^{-1}$ upon the transition to the 
FII form, and the line with a frequency of $1578 \mathrm{~cm}^{-1}$ is displaced by $12 \mathrm{~cm}^{-1}$, which is characteristic of the dome conformation. The drop in these frequencies is due to an increase in the bond length of methine bridges during complexation.

It should be emphasized that a planar molecular structure ( $\mathrm{F} 0$ form) formed mainly when molecules were deposited in inert gas matrices. Concentrations of the distorted $\mathrm{ZnP}$ forms in the Ar matrix were relatively small: the concentration of the FI form was less than 10\%, and that of the FII form was no more than $5 \%$. This testifies to the relatively weak disturbing effect the matrix has on the molecular structure of the metal complex.

The dome conformation is usually associated with the presence of extra ligands on the metal atom. The glow of only the FII form (see Fig. 1) is visible in the fluorescence spectrum when a small amount of pyridine (a strong extra ligand) is added to the $n$-octane, and there is no glow at all from the F0 and FI forms at a temperature of $4.2 \mathrm{~K}$.

It was established in [13] that the pyridine ligand attached to the metalloporphyrin in the hard matrix of the inert gases of metalloporphyrins breaks off during deposition, and no traces of it are recorded in the absorption spectrum. This confirms there is no initial liganding of the molecules embedded in the inert gas matrices and nitrogen matrices. Only relatively weak van der Waals complexes with gas atoms can form in matrices when preparing samples by means of isolation. This effect is not observed at room temperature, but the distance between the matrix (the inert gas) and a chromophore is less than one nanometer when the molecules are condensed into an inert gas matrix, which can greatly magnify the role of van der Waals complexes [14]. It should be considered that argon does not have its own dipole moment, but it does have fairly high polarizability (1.63 $\AA$ [15]). This leads to induction interactions, the magnitude of which for $\pi$ electron systems (aromatic compounds and porphyrins) can reach values of several $\mathrm{kcal} \cdot \mathrm{mol}^{-1}$ and will be maximal if the chromophore and matrix are located in parallel. As is described below, the complexing energies of metalloporphyrin with extraligands (the results from quantum chemical calculations) have comparable values and do not exceed $10 \mathrm{kcal} \mathrm{mol}^{-1}$ for $\mathrm{ZnP}$. 
Note too that the allowed vibrational structure has been recorded in the absorption spectrum (fluorescence excitation) of $\mathrm{ZnP}$ in a hard tetrahydrofuran matrix, in the region of the Soret band.

The spectrally resolved fluorescence excitation spectrum for the FI form of ZnP in solid tetrahydrofuran $(4.2 \mathrm{~K})$ in the region of the Soret band is shown in Fig. 4a. The same spectrum for the FII form of $\mathrm{ZnP}$ is correspondingly shown in Fig. 4b. We determined a number of normal vibration frequencies for the highlying electronic transitions of the Soret band. These were fully symmetrical vibrations with frequencies of 360, 719, 986, 1359, and $1477 \mathrm{~cm}^{-1}$ (Fig. 4). The fluorescence excitation spectra in the region of the Soret band had a pronounced doublet structure with different splitting values between the components when there were two spectral forms. The splitting value was almost $30 \%$ lower for the more distorted FII form of ZnP than for the FI form. From an empirical viewpoint, the reason for this is not entirely understood.
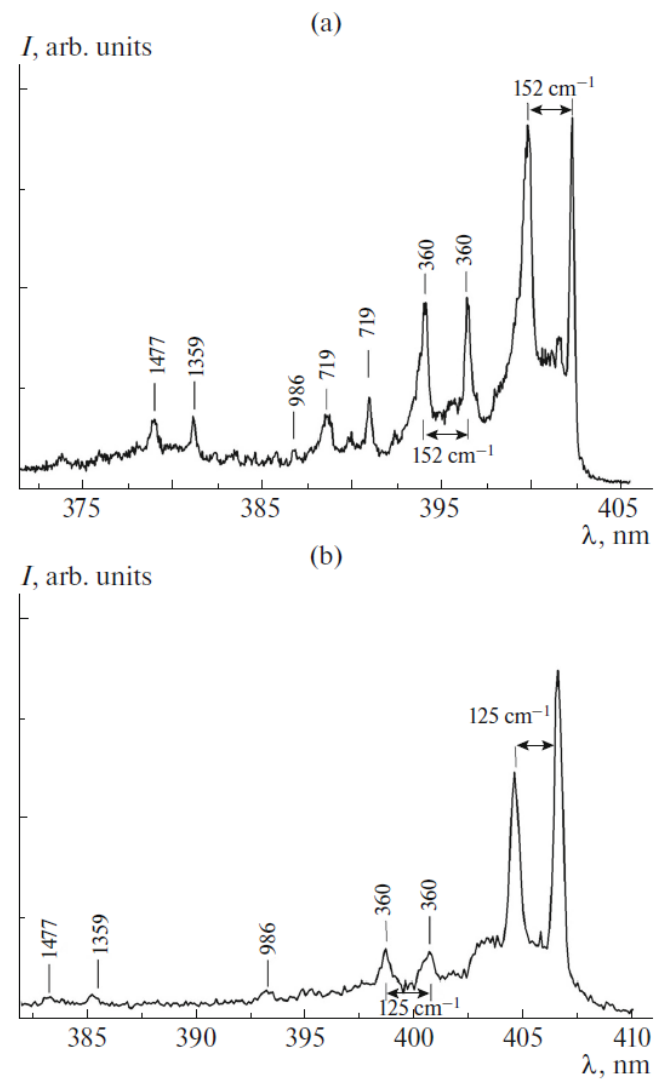

Fig. 4. Fluorescence excitation spectra of $\mathrm{ZnP}$ in the region of the Soret band for the FI form (a) at $\lambda_{\mathrm{fl}}=$ $562.7 \mathrm{~nm}$ and the FII form (b) at $\lambda_{\mathrm{fl}}=569.1 \mathrm{~nm}$ in a hard matrix of tetrahydrofuran at $4.2 \mathrm{~K}$.

Quantum chemical calculations (DFT and DFT with resolution in time) were performed; the ground and excited electronic states of the investigated metal complexes were modeled for a situation with the two 
forms of $\mathrm{MgP}$ and $\mathrm{ZnP}$ observed in solid tetrahydrofuran (THF). The results from experiments and theoretical calculations show that the formations of FI form with one ligand located above the metal atom, and of the FII form with two additional ligands at the top and bottom of the metal atom, are possible. The energy of complexing was this case estimated and proved to be $13.3 \mathrm{kcal} \mathrm{mol}^{-1}$ for $\mathrm{MgP}$ with one THF ligand, $18.3 \mathrm{kcal} \mathrm{mol}^{-1}$ for MgP with two THF ligands, $7.3 \mathrm{kcal} \mathrm{mol}^{-1}$ for $\mathrm{ZnP}$ with one THF ligand, and $8.5 \mathrm{kcal} \mathrm{mol}^{-1}$ for $\mathrm{ZnP}$ with two THF ligands.

\section{CONCLUSIONS}

The existence of different spectral forms in the luminescence spectra of metal complexes of porphyrins at low temperatures is of a fairly general nature.

There are two forms that have $0-0$ transition positions with a spectral shift of $360 \mathrm{~cm}^{-1}$ in the fluorescence spectra of $\mathrm{MgP}$ in the THF matrix at cryogenic temperatures, while the analogous shift in the THF matrix does not exceed $180 \mathrm{~cm}^{-1}$ for $\mathrm{ZnP}[9]$.

The existence of two spectral forms in the ground electronic state was observed in the phosphorescence spectra of the metal complexes of porphin with $\mathrm{Pd}(\mathrm{II})$ and $\mathrm{Pt}(\mathrm{II})$ when they were placed in the Shpol'skii matrix at a temperature of $4.2 \mathrm{~K}$. The energy gap for two spectral forms in the phosphorescence spectra of the porphin metal complex with the $\mathrm{Pd}(\mathrm{II})$ ion in the $n$-octane matrix at $4.2 \mathrm{~K}$ was $78 \mathrm{~cm}^{-1}$ [16], while the analogous energy gap for the $\mathrm{Pt}(\mathrm{II})$ complex of porphin had a much lower value: only $38 \mathrm{~cm}^{-1}$ [17]. Our results demonstrate the effectiveness of the fine-structural low-temperature spectroscopy of molecules in combination with modern quantum-mechanical ways of calculating the structure and spectral parameters of impurity molecular centers in low-temperature matrices for the study of structurally distorted macrocyclic compounds.

\section{ACKNOWLEDGMENTS}

This work was supported by the Belarus Foundation for Basic Research, project no. Ф16PA-007; and by the European Union as a part of the Horizon 2020 program, grant no. 645628. 


\section{REFERENCES}

1. Porphyrins and Metalloporphyrins, Smith, K.M., Ed., Amsterdam-New York-Oxford: Elsevier, 1975.

2. Solov'ev, K.N., Gladkov, L.L., Starukhin, A.S., et al., Spektroskopiya porfirinov: Kolebatel'nye sostoyaniya (Spectroscopy of Porphyrins: Vibrational States), Minsk: Nauka i Tekhnika, 1985.

3. Gorski, A., Starukhin, A., Stavrov, S., et al., Spectrochim. Acta A, 2017, vol. 173, p. 350.

4. Starukhin, A.S., Kruk, M.M., Gladkova, O.L., et al., Macroheterocycles, 2011, vol. 4, p. 85.

5. Cook, L.P., Brewer, G., and Wong-Ng, W., Crystals, 2017, vol. 7, p. 223.

6. Ishikawa, R., Horii, Y., Nakanishi, R., et al., Eur. J. Inorg. Chem., 2016, vol. 20, p. 3305.

7. Haddad, R., Gazeau, S., Pecaut, J., et al., J. Am. Chem. Soc., 2003, vol. 125, p. 1253.

8. Noort, M., Spectrochim. Acta, 1976, vol. 32, p. 747.

9. Starukhin, A.S. and Shul'ga, A.M., Opt. Spectrosc., 2005, vol. 98, p. 780.

10. Gorski, A., Knyukshto, V., Zenkevich, E., et al., J. Photochem. Photobiol., A, 2018, vol. 354, p. 101.

11. Zamiri, B., Reddy, K., Macgregor, R., et al., J. Biol. Chem., 2014, vol. 289, p. 4653.

12. Wasbotten, I.H., Conradie, J., and Ghosh, A., J. Phys. Chem. B, 2003, vol. 107, p. 3613.

13. Starukhin, A., Shulga, A., and Waluk, J., Chem. Phys. Lett., 1997, vol. 27, p. 405.

14. Barash, Yu.S. and Ginzburg, V.L., Sov. Phys. Usp., 1984, vol. 27, p. 467.

15. Meyer, B., Low Temperature Spectroscopy, New York: Elsevier, 1971.

16. Starukhin, A., Kruk, M., and Czerwieniec, R., J. Lumin., 2008, vol. 128, p. 531.

17. Starukhin, A. and Kruk, M., Macroheterocycles, 2009, vol. 2, p. 255. 